\title{
Morphological Changes of Conidiogenesis in Two Aspergillus Species
}

\author{
Ahmed Mohamed Aly Khalil ${ }^{1,2 *}$ and Amr Hosny Hashem ${ }^{2}$ \\ ${ }^{1}$ Biology Department, College of Science, Taibah University, Yanbu, Postal code 41911, KSA. \\ ${ }^{2}$ Botany and Microbiology Department, Faculty of Science, Al-Azhar University, Cairo - 13759, Egypt.
}

\begin{abstract}
Identification of aspergilli based on comparative of their diagnostic features in different growth media is a common manner between most of mycologists. Asexual sporulation in Aspergillus species is very complex processes which include numerous morphologically distinct stages. In the present study, asexual reproductive structures of Aspergillus terreus and Aspergillus versicolor have been tracked and the development of conidiophores have been compared in two types of growth media MEA (Malt Extract Agar) and YES (Yeast Extract Sucrose) which considered the most common growth media in identification of aspergilli. Interestingly, YES agar medium induce $A$. terreus to retard the asexual sporulation process in a distinctive manner. Unusually, the early conidiophore of $A$. terreus distends to form infertile (immature) vesicle which then produces a second conidiophore. The later conidiophore expands to form fertile (mature) vesicle which differentiates into conidial head. The development of one or more conidiophores from the immature vesicles of $A$. terreus instead the foot cells or areal hyphae remain mostly uncharacterized by many researchers. On the other hand, the asexual sporulation in both Aspergillus species was dramatically decreased on YES agar medium when compared by MEA medium. Moreover, the accessory conidia in A. terreus and reduced Penicillium-like structures that produce another type of phialedic conidia in $A$. versicolor were mostly not observed on YES agar medium.
\end{abstract}

Keywords: A. terreus, A. versicolor, asexual sporulation, scanning electron microscope.

\footnotetext{
*Correspondence: ahmed_khalil@azhar.edu.eg; Amsuliman@taibahu.edu.sa; 00966(0)580770171 Biology Department, College of Science, Taibah University, Yanbu, KSA.
}

(Received: 19 October 2018; accepted: 25 November 2018)

Citation: Ahmed Mohamed Aly Khalil and Amr Hosny Hashem, Morphological Changes of Conidiogenesis in Two Aspergillus Species, J Pure Appl Microbiol., 2018; 12(4):2041-2048. http://dx.doi.org/10.22207/JPAM.12.4.40

(c) The Author(s) 2018. Open Access. This article is distributed under the terms of the Creative Commons Attribution 4.0 International License which permits unrestricted use, sharing, distribution, and reproduction in any medium, provided you give appropriate credit to the original author(s) and the source, provide a link to the Creative Commons license, and indicate if changes were made. 


\section{INTRODUCTION}

Aspergillus species (collectively called aspergilli) are the most common saprophytic and/ or parasitic fungi that found in various climates worldwide. Most aspergilli reproduce asexually by forming conidiospores via a process called conidiogenesis (Axelrod, Gealt, \& Pastushok, 1973). Basically, conidia and conidial head structure are the most important morphological features that used by mycologists to identify and classify Aspergillus species into genera or distinguish closely related species. Although genetic approach have been interpreted in fungal taxonomy, phenotypic concepts are still necessary (Hyde, Abd-Elsalam, \& Cai, 2011).

Asexual reproductive cycle in aspergilli can be divided into two stages vegetative growth and sporulation development. The growth phase includes germination of a conidiospores and form a network of interconnected hyphae known as a mycelium. When the environmental factors changed, some of hyphae stop growing and initiate asexual reproduction by forming conidiophore and conidia (Adams, Wieser, \& Yu, 1998).

Moreover, the conidiogenesis process is influenced by environmental factors including carbon and nitrogen source, $\mathrm{pH}$, temperature, incubation period, and water potential (SITTON, 2004). One of the most important factors strikingly affecting morphological criteria in Aspergillus species is a composition of culture media (Khalil, 2016; Kim et al., 2005; Saha, Mandal, Dasgupta, \& Saha, 2008; Saxena, Sangeetha, Vohra, Gupta, \& Gulati, 2001). Malt extract agar and potato dextrose agar were the stander identification growth media for Aspergillus identification(Pitt, Hocking, \& Beuchat, 1998).

Furthermore, genetic regulations of the induction of conidia in fungi have been properly studied by many authors (Roncal \& Ugalde, 2003; Sun et al., 2012). However, several genes have been found to be involved in this process and controlled asexual sporulation(Roncal \& Ugalde, 2003; Sun et al., 2012; J.-W. Xu, Zhao, Xu, \& Zhong, 2012; Zhou, Wang, Qiu, \& Feng, 2012). Expectedly, environmental stresses have been found to act as regulatory factors for these genes(Chi \& Craven, 2016).

Accordingly, definite the effective environmental factors that induce asexual sporulation process in aspergilli considered the first step towards making an accurate identification. This paper present the morphological properties of Aspergillus terreus and Aspergillus versicolor including the conidiogenesis process when cultured on malt extract agar and yeast extract agar medium as a common medium used for identification of aspergilli.

\section{MATERIALS AND METHODS Microorganisms}

Aspergillus terreus (JCM 10227) and Aspergillus versicolor (NRRL 238) were kindly obtained from Culture Collection of school of Pharmacy and Bio-molecular science, Liverpool John Moores University, United Kingdom.

\section{Culture characteristics}

Culture characteristics of Aspergillus species were studied on two differential media, malt extract agar (MEA) HIMEDIA and yeast extract sucrose agar (YES) medium (2\% yeast extract (HIMEDIA), 15\% sucrose (HIMEDIA), and $1.5 \%$ agar (Sigma Aldrich)). Morphological characteristics including the diagnostic macroscopic features for identification such as colony diameter after ten days, color (conidia and reverse), exudates and colony texture were studied. Microscopic characteristics for the identification were conidial heads, stipes, color and length vesicles shape and seriation, metula covering, conidial size and shape were investigated.

\section{Bright field microscope}

Microscopic examination was carried out by tearing small apart of mycelium in a droplet of $0.1 \%$ lactophenol blue or by cover slip culture technique which designated by(Nugent, Sangvichen, Sihanonth, Ruchikachorn, \& Whalley, 2006) and the preparation were examined by bright field microscopy built in with camera using $\times 40$ and $\times 60$.

\section{Scanning electron microscope}

Small pieces from the fresh colony of Aspergillus culture were fixed in $2.5 \%$ glutaraldehyde for 20 minutes to provide a rapid inter and intra-cellular penetration. Fixed specimens were dehydrated by series concentrations of ethanol, ending with $100 \%$ dehydrating liquid in highest purity. Typically, concentrations are steps of $10,20,30,50,70,90$, $95,100 \%$ at 10 minutes for each, with 3 changes at 
$100 \%$. Acetone was used as the intermediate fluid because it is miscible with carbon dioxide. Critical point drying (CPD) was utilized to avoid collapse in SEM. This step is carried out by substituting acetone with liquid $\mathrm{CO}_{2}$ then the liquid $\mathrm{CO}_{2}$ is kept at a critical temperature and pressure $\left(34.5^{\circ} \mathrm{C}\right.$ and 1200 psi (pounds per square inch)). Eventually, samples were coated with gold using an Emitech K550X coating machine. The specimens were then loaded into FEI (Quanta 200) ESEM (Environmental Scanning Electron Microscopy, 2008) and observed over a range of magnifications. Images were got by using an image capture system (Oxford Instruments, INCA system, Oxford, UK).

\section{Counting of fungal spores}

Counting of fungal spores was achieved bytaking $9 \mathrm{~mm}$ disc of fungal cultures into $10 \mathrm{ml}$ test tube filled with $5 \mathrm{ml}$ of distilled water. Test tubes were shacked vigorously by vortex mixer for $5 \mathrm{~min}$. Ten micrometer of fungal spores suspension was transferred into a petroffhausser counting chamber and then spores was counted. Consequently, the number of fungal spores in 5 $\mathrm{ml}$ was calculated.

\section{RESULTS AND DISCUSSION}

Colonies of $A$. terreus on MEA reaching $50 \mathrm{~mm}$ in diameter after incubating for ten days at $25^{\circ} \mathrm{C}$. Surface colors are likely brownish to buff and reverse dark yellow to brown (Fig. 1). Conidial heads on MEA densely columnar, 70-150 $\mu \mathrm{m}$ in length $40-50 \mu \mathrm{m}$ in diameter. Conidiophore smooth to slightly rough, hyaline, 150-250 $\mathrm{mm}$ in length and 5-6 $\mu \mathrm{m}$ wide. Vesicle biseriates, hemispherical to globose, $15-20 \mu \mathrm{m}$ in diameter (Table 1). Metulae flask shape, covering upper $2 / 3$ of the vesicle, $4.5-7.5 \times 2.0-3.5 \mu \mathrm{m}$; phialides $5.5-8.0 \times 1.5-3 \mu \mathrm{m}$. Conidia globose to subglobose, $2-3 \mu \mathrm{m}$ in diameter (Figs. 1\&3). Usually, growth of $A$. terreus on MEA follows the same way of other Aspergillus species. The vegetative growth initiated by the germination of conidiospores which lead to the formation of tubular hyphae that grow and branch to form mycelium. The mycelium expands and grows until environmental conditions are divers. At this time, aerial hyphal are rising up in the center of the colony and some of them successively differentiate into conidiophores (Adams et al., 1998).

On the other hand, colonies of $A$. terreus on YES agar plate reach $70 \mathrm{~mm}$ in diameter after incubating for ten days at $25^{\circ} \mathrm{C}$. Surface colors be likely whitish and when it's get old changing to yellow in the center of colony while the reverse color pale yellow (Fig.1). Two conidiophores are formed, one from the foot cells and the other
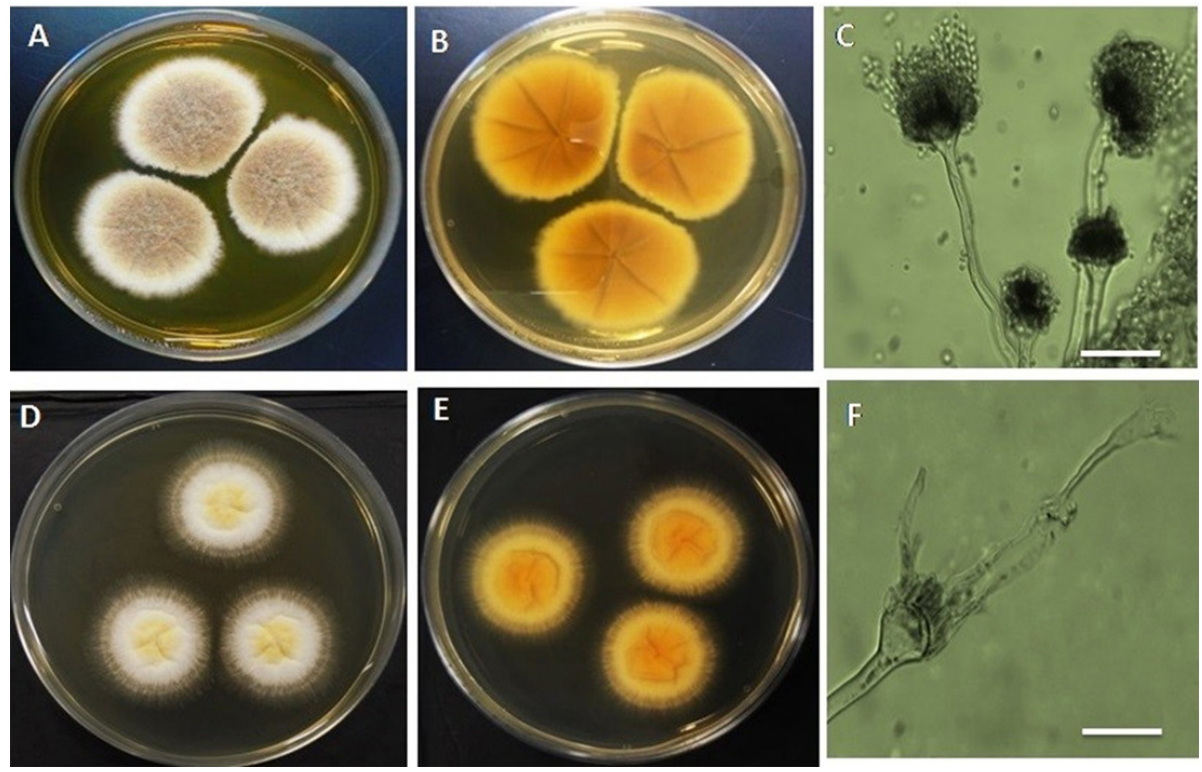

Fig. 1. Aspergillus terrues A\&B. Surface and reverse on MEA C. conidiophore with conidial head D\&E. Surface and reverse on YES agar F. Conidiophores with immature vesicle (Bar in C \& F = 100 $\mu \mathrm{m}$ ). 
Khalil \& Hashem J Pure App/ Microbiol, 12(4), 2041-2048 | Dec. 2018 | http://dx.doi.org/10.22207/JPAM.12.4.40

Table 1. Culture characteristics of $A$. terreus in two common growth media MEA and YES agar

\begin{tabular}{|c|c|c|}
\hline Characters & Examination on MEA & Examination on CYA \\
\hline \multicolumn{3}{|l|}{ Culture examination } \\
\hline Growth characteristics & $\begin{array}{l}\text { Colonies grow moderate to } \\
\text { rapidly reaching } 2.0-5.0 \mathrm{~cm} \\
\text { diameter in } 10 \text { days at } 25^{\circ} \mathrm{C} \text {, } \\
\text { cinnamon to light brown, buff } \\
\text { colonies. } \\
\text { Reverse light brown. }\end{array}$ & $\begin{array}{l}\text { Colonies grow moderate to } \\
\text { rapidly reaching } 3.0-7.0 \mathrm{~cm} \\
\text { diameter in } 10 \text { days at } 25^{\circ} \mathrm{C} \text {, } \\
\text { white colonies with yellow } \\
\text { pigment in center. } \\
\text { Reverse light brown. }\end{array}$ \\
\hline \multicolumn{3}{|c|}{ Microscopic examination: } \\
\hline Conidial heads & Columnar. & Hemisphere \\
\hline Conidiophore & 5.0-6.0 $\mu \mathrm{m}$ in diameter. & $\begin{array}{l}4.0-6.0 \mu \mathrm{m} \text { in diameter. Two } \\
\text { conidiophores have the same } \\
\text { size }\end{array}$ \\
\hline Vesicle & $\begin{array}{l}\text { Globose to subglobose shape. } \\
15.0-20.0 \mu \mathrm{m} .\end{array}$ & $\begin{array}{l}\text { Both vesicles are globose to } \\
\text { subglobose. } \\
\text { fertile one } 10.0-15.0 \mu \mathrm{m} \text {. } \\
\text { infertile } \\
\text { vesicle } 7.0-10.0 \mu \mathrm{m}\end{array}$ \\
\hline Matula and phialides & In two series (biseriate) & In two series (biseriate) \\
\hline Conidia & Conidia, spherical, 2.0-3.0 $\mu \mathrm{m}$. & Conidia, spherical, 2.0-3.0 $\mu \mathrm{m}$. \\
\hline $\begin{array}{l}\text { Sporulation No. of } \\
\text { spores } / \mathrm{cm}^{2}\left(\times 10^{5}\right)\end{array}$ & 75 & 40 \\
\hline
\end{tabular}

come out of the vesicle. Both conidiophores are mostly same in the length approximately 100$120 \mu \mathrm{m}$. The first immature vesicle is globose and smaller than the second one $7-10 \mu m$ (Table 1). The second vesicle is mature and produces matula and phialides exactly as this in MEA (Figs. 1\&3).
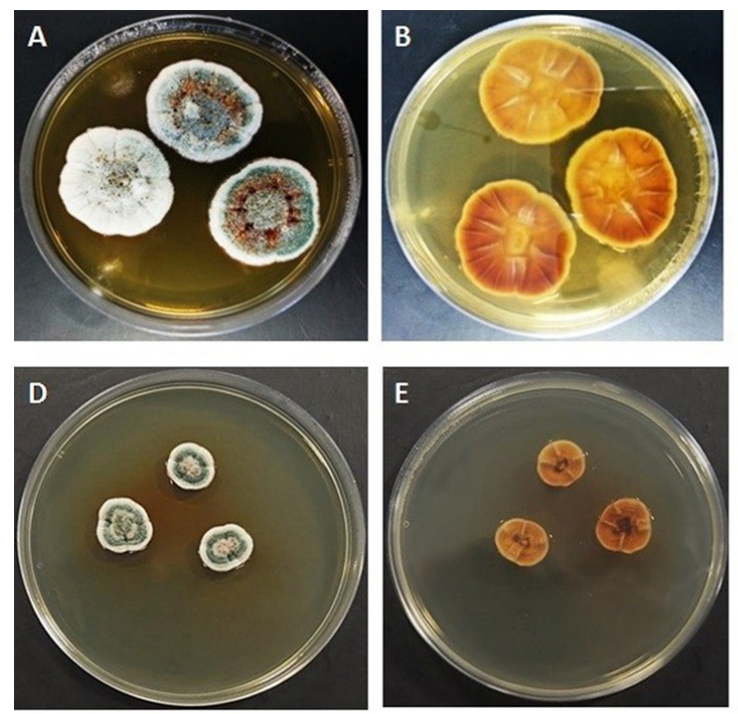

30-35 $\mathrm{mm}$ in diameter after incubating for ten days at $25^{\circ} \mathrm{C}$. Colony surface was light to dark greenish to bluish green color while reverse was light brown to orange. Moreover, colonies of $A$. versicolor on YES agar medium reaching 20-25
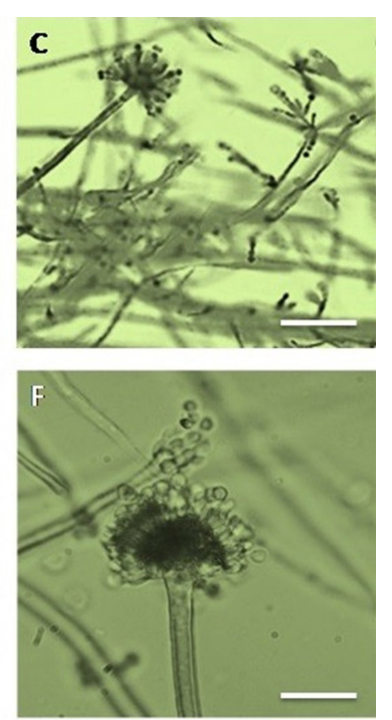

Fig. 2. Aspergillus vericolor A \& B. Surfaceand reverse on MEA C. Two pattern of coniophores (aspergillate and penicillate structures) D\&E. Surface and reverse on YES agar F. Conidiophore with conidial head (Bar in C \& F $=400 \mu \mathrm{m}$ ).

Journal of Pure and Applied Microbiology

2044

www.microbiologyjournal.org 
$\mathrm{mm}$ in diameter after incubating for ten days at $25^{\circ} \mathrm{C}$ (Fig. 2). Colony surface was green to greenish while the reverse was reddish brown in color. In both growth media, conidiophores elongate and swell to form fertile vesicle with smooth walled stipes 200-700 5-7.0 $\mu \mathrm{m}$. Vesicles are spherical to sub-spherical, or pyriform to spatulate, $8-15 \mu \mathrm{m}$ in diameter. Conidial heads biseriate, Phialides

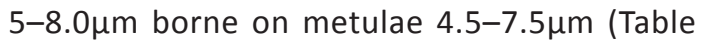
2 ). Both phialidic conidia which produced from conidial head and penicillium like structure are globose to sub globose with roughened wall (Figs. 2, 4 \& 5).

Consequently, the nutrient level of YES agar medium is much sufficient for $A$. terreus than that in MEA. Thus, many researchers stated YES to be the best media for hyphal growth (Maheswari, Singh, \& Sahu, 1999; Saha et al., 2008; Suzuki \& Iwahashi, 2016; S. Xu, Yuan, \& Chen, 1984).

Whereas, sucrose is one of the most obvious different between YES and MEA, it plays an important role in sporulation process(Latha, Prakasam, Jonathan, Samiyappan, \& Natarajan, 2013). Fungi on glucose free growth medium produce the high number of conidia. This is probably due to low sugar concentration. Moreover, vegetative growth in some fungal species was stimulated in vitro by the restoration of the medium with sugar(Latha et al., 2013).

In fact, when sufficient nutrients are available and environmental conditions are favorable $A$. terreus sustained in vegetative growth. However, when conditions diverse $A$. terreus tends to initiate sporulation process through forming aerial hypha which then differentiate to conidiophore and terminate by vesicle. Continuously, on MEA vesicle produces simultaneous matula and phialide (all matula on the surface of vesicle developed in the same time). Eventually, Phialides sprout chains of conidiospores which germinate when nutrient is available to produce germ tube and vegetative hyphyae once again. Moreover, some researchers recorded that, A. terreus has ability to produce microconidia borne directly on a short conidiophores which arise laterally from vegetative hyphae(Walsh et al., 2003).

Interestingly, when $A$. terreus cultured on high rich sucrose medium such as YES agar the first conidiophore that differentiate directly from areal hyphae produce infertile vesicle. After a short of time, this vesicle is simultaneously fully covered by matula like structure. One or two of them are differentiated into conidiophores which expand to form the second fertile vesicle.

Table 2. Culture characteristics of $A$. versicolor in two common growth media MEA and YES agar

\begin{tabular}{|c|c|c|}
\hline Characters & Examination on MEA & Examination on CYA \\
\hline \multicolumn{3}{|l|}{ Culture examination } \\
\hline \multirow[t]{3}{*}{ Growth characteristics } & $\begin{array}{l}\text { Colonies grow rapidly } \\
\text { in } 10 \text { days at } 25^{\circ} \mathrm{C} \text {, greenish to } \\
\text { reaching } 3.0-3.5 \mathrm{~cm} \text { diameter }\end{array}$ & $\begin{array}{l}\text { Colonies grow moderate to } \\
\text { rapidly reaching } 2.0-\end{array}$ \\
\hline & $\begin{array}{l}2.5 \mathrm{~cm} \text { diameter in } 10 \\
\text { blue green colonies. }\end{array}$ & $\begin{array}{l}\text { days at } 25^{\circ} \mathrm{C} \text {, greenish to } \\
\text { blue green colonies. }\end{array}$ \\
\hline & Reverse light brown to orange. & $\begin{array}{l}\text { Reverse light brown to } \\
\text { reddish }\end{array}$ \\
\hline \multicolumn{3}{|l|}{ Microscopic examination } \\
\hline Conidial heads & pyriform to spatulate & pyriform to spatulate \\
\hline Conidiophore & $5.0-7.0 \mu \mathrm{m}$ in diameter. & 5.0-7.0 $\mu \mathrm{m}$ in diameter. \\
\hline Vesicle & $\begin{array}{l}\text { Globose to subglobose shape. } \\
8.0-15.0 \mu \mathrm{m}\end{array}$ & $\begin{array}{l}8.0-15.0 \mu \mathrm{m} \text {. Globose } \\
\text { subglobose shape. }\end{array}$ \\
\hline Matula and phialides & In two series (biseriate) & In two series (biseriate) \\
\hline Conidia & $\begin{array}{l}\text { Roughglobose to sub- } \\
\text { globose, } 2.0-3.0 \mu \mathrm{m} \text {. }\end{array}$ & $\begin{array}{l}\text { Roughglobose to sub- } \\
\text { globose, } 2.0-3.0 \mu \mathrm{m}\end{array}$ \\
\hline $\begin{array}{l}\text { Sporulation No. of } \\
\text { spores } / \mathrm{cm}^{2}\left(\times 10^{5}\right)\end{array}$ & 85 & 70 \\
\hline
\end{tabular}


To this end, the fertile vesicle produce matula and phialide and then conidia are formed in chains. Moreover, the accessory conidia in $A$. terreus have been observed on MEA medium while it is mostly disappeared on YES agar medium (Fig. 5). Probably, accessory conidia in A. terreus appeared to be more susceptible to high sugar concentrations in growth media(Deak, Wilson, White, Carr, \& Balajee, 2009).
Based on our knowledge, no one has been reported vesicle of $A$. terreus or any other Aspergillus species can sprout one or more conidiophores instead matula or phialides. Even mutant strains of $A$. nidulans and $A$. fumigatus which designated to inhibit sporulation process and carry on vegetative reproduction, they only produce abnormal elongated conidiophore with or without conidia from its vesicle (Adams et
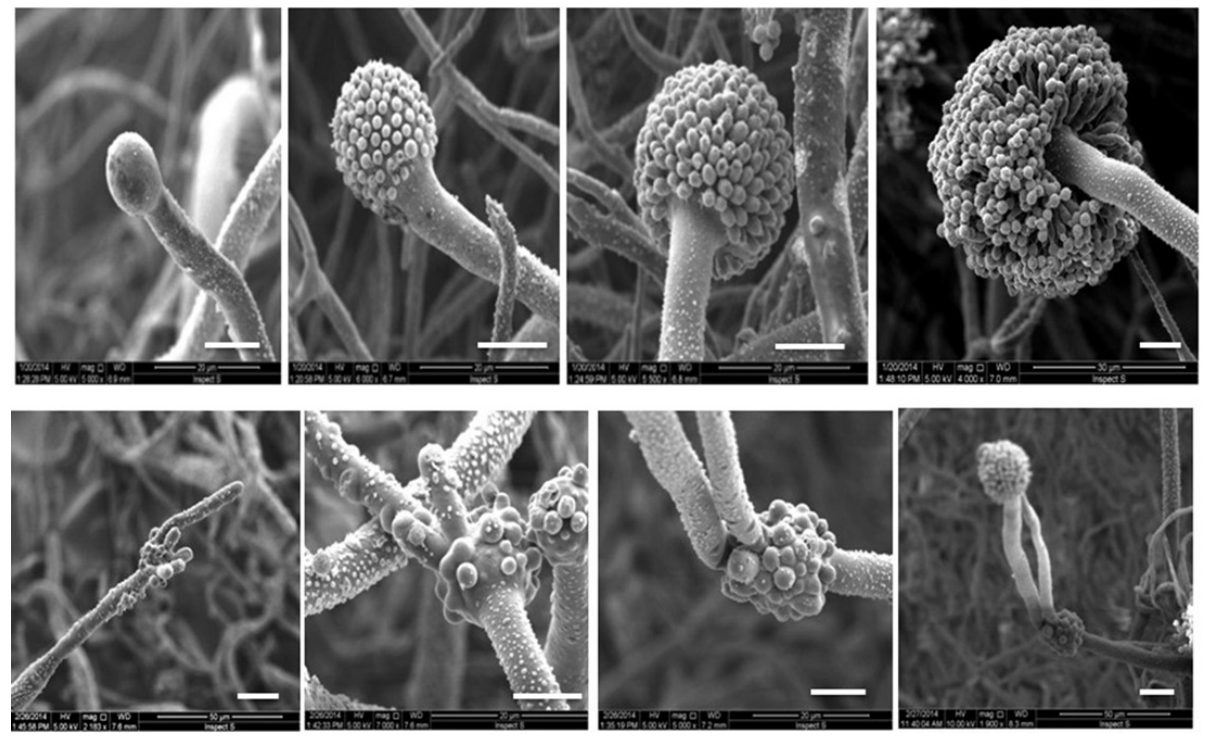

Fig. 3. Scanning electron micrographs of Aspergillus terreus. The top row shows the formation of conidiophore and conidial head on MEA medium. The bottom row shows the formation of conidiophores and the fertile and infertile vesicles on YES agar medium (Bars $=10 \mu \mathrm{m})$.
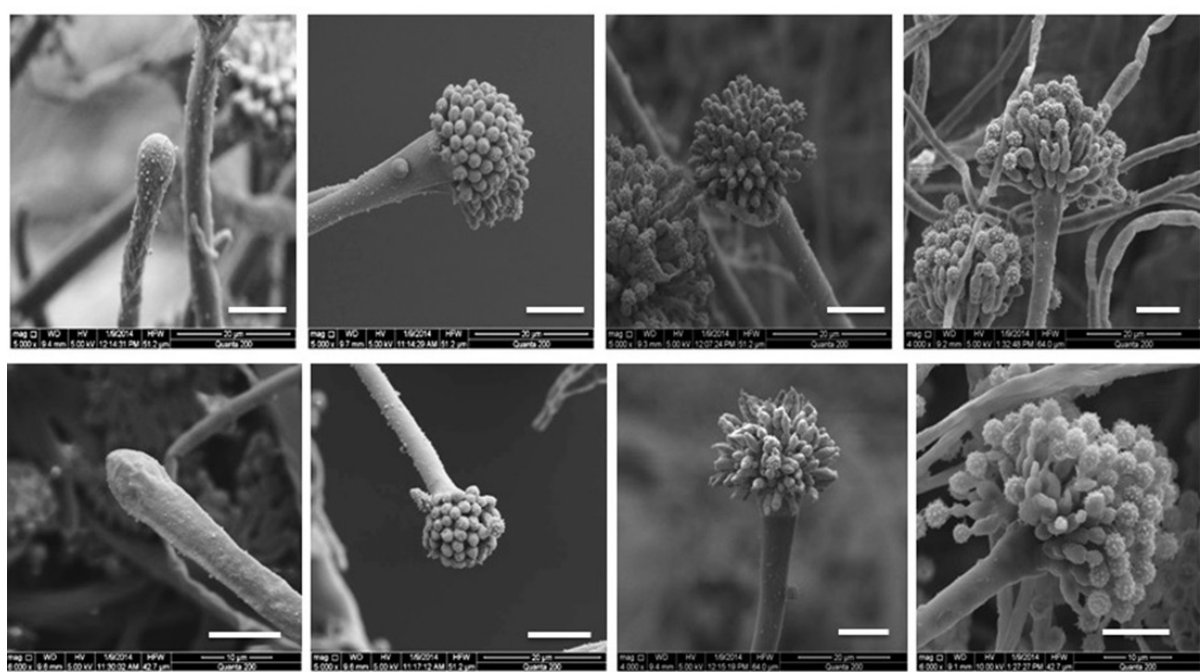

Fig. 4. Scanning electron micrographs of Aspergillus versicolor. The top row shows the formation of conidiophore and conidial head on MEA medium. The bottom row shows the formation of conidiophore and conidial head on YES agar medium (Bars $=10 \mu \mathrm{m})$. 

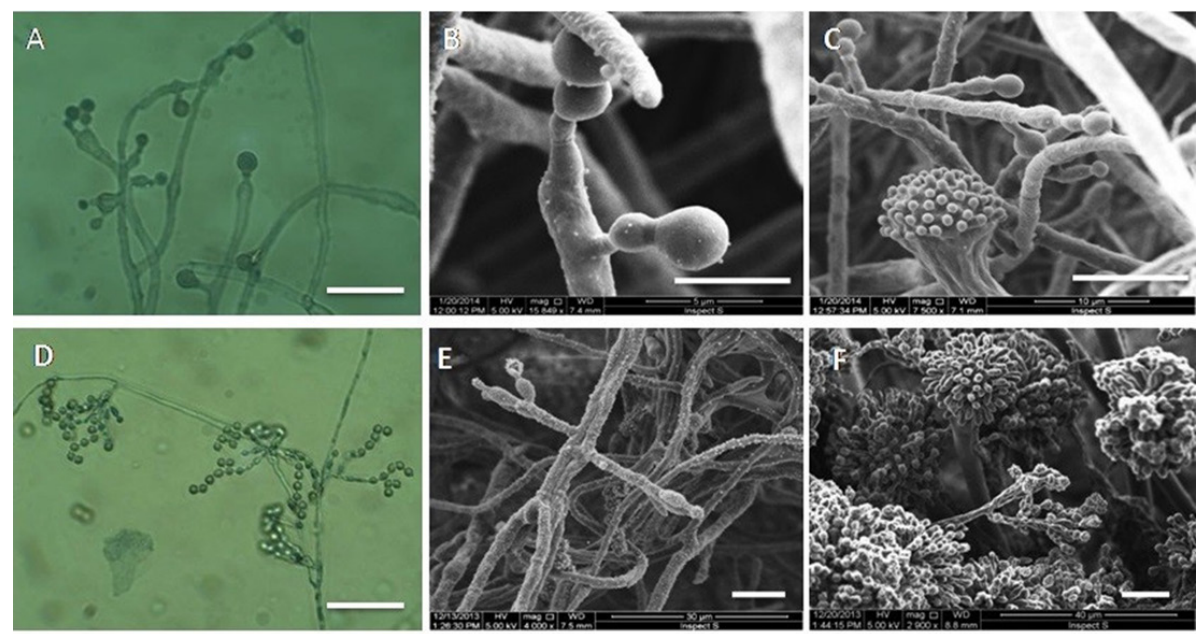

Fig. 5. Bright field and scanning electron micrographs. Top row shows the accessory conidia in Aspergillus terreus grown on MEA. Bottom row shows the phialidic conidia on penicillium like structure in Aspergillus versicolor on MEA (Bars: $A$ \& $D=400 \mu \mathrm{m} ; B=5 \mu \mathrm{m} ; C, E, \& F=10 \mu \mathrm{m}$ ).

al., 1998; Krijgsheld et al., 2013; Ni, Gao, Kwon, Shin, \& Yu, 2010). It believes that, some genes induced by high concentration of sugar to increase the vegetative growth and delay sporulation process(Krijgsheld et al., 2013). In this case, suppression of sporulation process occur when fungus sustaining the mycelium growth while aerial hyphae if it is formed do not differentiate into conidiophore and conidial head(Adams et al., 1998).

In our point of view, $A$. terreus might be found another or more than one way to delay the sporulation process. In addition to the previous way, the aerial hypha differentiates into conidiophores and immature vesicle which subsequently produce one or more additional conidiophores and vesicles before starting in actual sporulation process.

Obviously, the vegetative growth of A. versicolor on MEA was much faster and greater than that on YES agar. Contrary, asexual sporulation on MEA was much higher than that on YES agar. However, conidiogenous structures of $A$. terreus showed no variations on two growth media except the absence of penicillium like structures on YES agar medium. It has also been assumed that the phialidic conidia that produced by penicillium like structures might be inhibited by sucrose in YES agar medium (Khalil, 2016).

In conclusion, the taxonomic criteria should be taken in concern when culturing and identifying $A$. terreus on any growth medium contain high sugar concentration specially YES agar medium. Moreover, it is useful to use high rich sugar medium in biotechnology where sporulation processes undesired during this application. Furthermore, SEM represents a precious investigative tool to examine and characterize any newly morphological changes that occur to fungi as environmental conditions alter.

\section{ACKNOWLEDGMENTS}

I'd like to show my gratitude to emeritus professor Anthony Whalley in Liverpool John Moores University for providing me the organisms which have been used in this work.

\section{CONFLICT OF INTEREST}

The author report no conflicts of interest. The author alone is responsible for the content and writing of the paper.

\section{REFERENCES}

1. Adams, T. H., Wieser, J. K., \& Yu, J.-H. Asexual sporulation in Aspergillus nidulans. Microbiology and molecular biology reviews, 1998; 62(1), 3554.

2. Axelrod, D., Gealt, M., \& Pastushok, M. Gene control of developmental competence in Aspergillus nidulans. Developmental biology, 1973; 34(1), 9-15.

3. Chi, M.-H., \& Craven, K. D. RacA-mediated 
ROS signaling is required for polarized cell differentiation in conidiogenesis of Aspergillus fumigatus. PloS one, 2016; 11(2), e0149548.

4. Deak, E., Wilson, S. D., White, E., Carr, J. H., \& Balajee, S. A. Aspergillus terreus accessory conidia are unique in surface architecture, cell wall composition and germination kinetics. PloS one, 2009; 4(10), e7673.

5. Hyde, K. D., Abd-Elsalam, K., \& Cai, L. Morphology: still essential in a molecular world. Mycotaxon, 2011; 114(1), 439-451.

6. Khalil, A. Variation in conidiophore complexity in Aspergillus versicolor. Current Research in Environmental \& Applied Mycology, 2016; 6(3), 150-158.

7. Kim, J. H., Campbell, B. C., Yu, J., Mahoney, N., Chan, K. L., Molyneux, R. J., . . . Cleveland, T. E. Examination of fungal stress response genes using Saccharomyces cerevisiae as a model system: targeting genes affecting aflatoxin biosynthesis by Aspergillus flavus Link. Applied Microbiology and Biotechnology, 2005; 67(6), 807-815.

8. Krijgsheld, P., Bleichrodt, R. v., Van Veluw, G., Wang, F., Müller, W., Dijksterhuis, J., \& Wösten, $\mathrm{H}$. Development in Aspergillus. Studies in mycology, 2013; 74, 1-29.

9. Latha, P., Prakasam, V., Jonathan, E., Samiyappan, R., \& Natarajan, C. Effect of culture media and environmental factors on mycelial growth and pycnidial production of Lasiodiplodia theobromae in physic nut (Jatropha curcas). Journal of environmental biology, 2013; 34(4), 683.

10. Maheswari, S., Singh, D., \& Sahu, A. Effect of several nutrient media on the growth and sporulation of Alternaria alternata. Journal of Mycopathological Research, 1999; 37(1), 21-24.

11. Ni, M., Gao, N., Kwon, N.-J., Shin, K.-S., \& Yu, J.-H. Regulation of Aspergillus conidiation Cellular and Molecular Biology of Filamentous Fungi, 2010; (pp. 559-576): American Society of Microbiology.

12. Nugent, L. K., Sangvichen, E., Sihanonth, P., Ruchikachorn, N., \& Whalley, A. J. A revised method for the observation of conidiogenous structures in fungi. mycologist, 2006; 20(3), 111-114.

13. Pitt, J., Hocking, A., \& Beuchat, L. R. Fungi and food spoilage (2nd edn). Trends in Food Science and Technology, 1998, 9(2), 89.
14. Roncal, T., \& Ugalde, U. Conidiation induction in Penicillium. Research in Microbiology, 2003; 154(8), 539-546.

15. Saha, A., Mandal, P., Dasgupta, S., \& Saha, D. Influence of culture media and environmental factors on mycelial growth and sporulation of Lasiodiplodia theobromae (Pat.) Griffon and Maubl. Journal of environmental biology, 2008; 29(3), 407.

16. Saxena, R., Sangeetha, L., Vohra, A., Gupta, R., \& Gulati, R. Induction and mass sporulation in lignin degrading fungus Ceriporiopsis subvermispora for its potential usage in pulp and paper industry. Current Science, 2001; 591-594.

17. SITTON, J. W. Effects of culture media and environmental factors on mycelial growth and pycnidial production of Potebniamyces pyri. Mycological research, 2004; 108(8), 926-932.

18. Sun, X., Yu, L., Lan, N., Wei, S., Yu, Y., Zhang, H., . Li, S. Analysis of the role of transcription factor VAD-5 in conidiation of Neurospora crassa. Fungal genetics and biology, 2012; 49(5), 379387.

19. Suzuki, T., \& Iwahashi, Y. Addition of Carbon to the Culture Medium Improves the Detection Efficiency of Aflatoxin Synthetic Fungi. Toxins, 2016; 8(11), 338.

20. Walsh, T. J., Petraitis, V., Petraitiene, R., FieldRidley, A., Sutton, D., Ghannoum, M., ... Bacher, J. Experimental pulmonary aspergillosis due to Aspergillus terreus: pathogenesis and treatment of an emerging fungal pathogen resistant to amphotericin B. The Journal of infectious diseases, 2003; 188(2), 305-319.

21. Xu, J.-W., Zhao, W., Xu, Y.-N., \& Zhong, J.-J. Isolation and analysis of differentially expressed genes during asexual sporulation in liquid static culture of Ganoderma lucidum by suppression subtractive hybridization. Molecular biology reports, 2012; 39(4), 3603-3610.

22. Xu, S., Yuan, S., \& Chen, X. Studies on pathogenic fungus (Alternaria tennuis Nees) of poplar leaf blight. J. North East For. Inst, 1984; 12, 56-64.

23. Zhou, G., Wang, J., Qiu, L., \& Feng, M. G. A Group III histidine kinase (mhk1) upstream of high osmolarity glycerol pathway regulates sporulation, multi stress tolerance and virulence of Metarhizium robertsii, a fungal entomopathogen. Environmental microbiology, 2012; 14(3), 817-829. 